\title{
A new all-round density functional based on spin states and $S_{N} 2$ barriers
}

\author{
Marcel Swart, ${ }^{1,2, a)}$ Miquel Solà, ${ }^{1}$ and F. Matthias Bickelhaupt ${ }^{3}$ \\ ${ }^{1}$ Departament de Química and Institut de Química Computacional, Universitat de Girona, \\ Campus Montilivi, 17071 Girona, Catalunya, Spain \\ ${ }^{2}$ Institució Catalana de Recerca i Estudis Avançats (ICREA), Pg. Lluís Companys 23, \\ 08010 Barcelona, Catalunya, Spain \\ ${ }^{3}$ Department of Theoretical Chemistry and Amsterdam Center for Multiscale Modeling, \\ Vrije Universiteit Amsterdam, De Boelelaan 1083, 1081 HV Amsterdam, The Netherlands
}

(Received 20 May 2009; accepted 7 August 2009; published online 1 September 2009)

\begin{abstract}
We report here a new empirical density functional that is constructed based on the performance of OPBE and PBE for spin states and $\mathrm{S}_{\mathrm{N}} 2$ reaction barriers and how these are affected by different regions of the reduced gradient expansion. In a previous study [Swart, Solà, and Bickelhaupt, J. Comput. Methods Sci. Eng. 9, 69 (2009)] we already reported how, by switching between OPBE and PBE, one could obtain both the good performance of OPBE for spin states and reaction barriers and that of PBE for weak interactions within one and the same (SSB-sw) functional. Here we fine tuned this functional and include a portion of the KT functional and Grimme's dispersion correction to account for $\pi-\pi$ stacking. Our new SSB-D functional is found to be a clear improvement and functions very well for biological applications (hydrogen bonding, $\pi-\pi$ stacking, spin-state splittings, accuracy of geometries, reaction barriers). (C) 2009 American Institute of Physics. [doi:10.1063/1.3213193]
\end{abstract}

\section{INTRODUCTION}

Over the past 20 years, density functional theory $(\mathrm{DFT})^{1-3}$ has become the method of choice for computational studies in organic chemistry, organometallics, and biochemistry. This is mainly due to the combination of general accuracy and high efficiency, which enables one to treat real molecular systems at relatively low computational cost. Within Kohn-Sham DFT, only the exchange-correlation energy $\left(E_{X C}\right)$ needs to be approximated, for which many formulations have been proposed. The earliest DFT functionals took only the density $\rho(r)$ into consideration [local density approximations (LDAs)], which was observed to be a big improvement over Hartree-Fock. Further improvements were obtained by taking into account more and more density ingredients [see Eq. (1)], such as the density gradient $\nabla \rho$ [in generalized gradient approximations, (GGAs) $],{ }^{4}$ and its Laplacian $\nabla^{2} \rho$ and/or the kinetic-energy density $\tau$ (in metaGGA or MGGA functionals; Perdew and Constantin recently showed $^{5}$ that these latter two ingredients carry essentially the same information beyond the density and density gradient). ${ }^{6,7}$

$E_{X C}^{\mathrm{LDA}}\left[\rho_{\alpha}, \rho_{\beta}\right]=\int d^{3} r \varepsilon_{X C}^{\mathrm{unif}}\left(\rho_{\alpha}, \rho_{\beta}\right)$,

$E_{X C}^{\mathrm{GGA}}\left[\rho_{\alpha}, \rho_{\beta}\right]=\int d^{3} r f\left(\rho_{\alpha}, \rho_{\beta}, \nabla \rho_{\alpha}, \nabla \rho_{\beta}\right)$,

$E_{X C}^{\mathrm{MGGA}}\left[\rho_{\alpha}, \rho_{\beta}\right]=\int d^{3} r f\left(\rho_{\alpha}, \rho_{\beta}, \nabla \rho_{\alpha}, \nabla \rho_{\beta}, \nabla^{2} \rho_{\alpha}, \nabla^{2} \rho_{\beta}, \tau_{\alpha}, \tau_{\beta}\right)$.

\footnotetext{
a) Author to whom correspondence should be addressed. Electronic mail: marcel.swart@icrea.es.
}

Perdew et al. ${ }^{7,8}$ used these ingredients in order to build Jacob's ladder of density functional approximations, where each rung is built around those prior to it and with increasing proximity to the heaven of chemical accuracy. Although the systematic and nonempirical approach taken by Perdew indeed seems to lead to constant improvement, there does not seem to be a systematic improvement for all properties. This can be directly traced back to the process of solving the Schrödinger equation $H \Phi=E \Phi$. In contrast with wave function theory that systematically improves the wave function $\Phi$ (but not necessarily all molecular properties), density functional methods tinker with the Hamiltonian $H$. As a result, no systematic improvement can be expected within DFT (although Perdew's nonempirical series of functionals comes very close) and every new density functional has to be validated for its appropriateness for different properties and characteristics.

We recently validated a large number of density functionals for either spin states, ${ }^{9,10}$ hydrogen bonding, ${ }^{11} \pi-\pi$ stacking, ${ }^{12}$ accuracy of geometries, ${ }^{13}$ and $\mathrm{S}_{\mathrm{N}} 2$ reaction barriers. ${ }^{14-17}$ From these studies, it emerged that Perdew's GGA functional (PBE) ${ }^{18,19}$ performs very well in many aspects, but also fails dramatically for others. Interestingly enough, for those interactions for which PBE fails (spin states, $\mathrm{S}_{\mathrm{N}} 2$ barriers), ${ }^{9,15}$ it helps to replace the PBE exchange part with Handy and Cohen's OPTX (Ref. 20) functional [to give OPBE (Ref. 14)]. Instead, for those interactions that PBE works very well (hydrogen bonding) or reasonably well ( $\pi-\pi$ stacking), OPBE fails. ${ }^{11,12}$ We attempted to rationalize what causes this different behavior, which was initially attributed to the inclusion of $s^{4}$ terms [where $s$ is a reduced density gradient, $\left.s=|\nabla \rho| /\left(2\left(3 \pi^{2}\right)^{1 / 3} \rho^{4 / 3}\right)\right]$ in the OPTX functional. ${ }^{9}$ We previously studied what was the influence of 


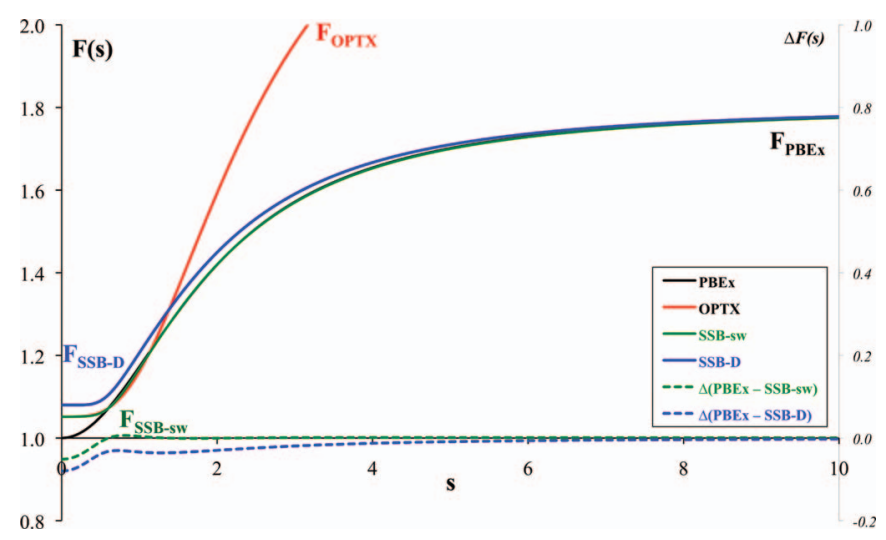

FIG. 1. Enhancement factors for OPTX, PBE, SSB-sw, and SSB-D exchange functionals and differences between PBE and the SSB functionals.

the constraints put on the PBE exchange functional by Perdew, but did not find any clear correlation with the performance for different interactions. $^{21}$

An important step forward was made recently ${ }^{22}$ when we investigated which part of the (exchange) functional is responsible for which interactions. In that study we exploited the fact that the only difference between PBE and OPBE is in the formulation of the exchange functional. This allowed for the design of a series of functionals that switch smoothly from OPBE to PBE at a predefined point $P$ of the reduced density gradient $s$, where $P$ runs from $s=0.1$ to $s=10.0$ :

$$
\begin{aligned}
& q_{\mathrm{sw}}(s)=1 \quad s<a, \\
& q_{\mathrm{sw}}(s)=\frac{(b+2 s-3 a)(b-s)^{2}}{(b-a)^{3}}, \quad a<s<b, \\
& q_{\mathrm{sw}}(s)=0 \quad s>b \\
& a=P-0.1, \quad b=P+0.1 .
\end{aligned}
$$

The enhancement factor for the switched exchange functional then has the form:

$$
F_{\mathrm{sw}}(s)=q_{\mathrm{sw}}(s) \cdot F_{\mathrm{OPTX}}(s)+\left(1-q_{\mathrm{sw}}(s)\right) \cdot F_{\mathrm{PBEX}}(s) .
$$

We observed in that study that for a number of properties the different behaviors of OPBE versus PBE is completely determined by one particular region, i.e., the region of $s<1$. That is, the excellent behavior of OPBE for spin states and the barriers of $\mathrm{S}_{\mathrm{N}} 2$ reactions can be obtained with a $P$ value of ca. 0.7-0.9, without any deterioration of the PBE results for hydrogen bonding or $\pi-\pi$ stacking. Based on these data, we designed a small modification of the PBE exchange functional in order to mimic these findings:

$$
F_{\text {SSB-sw }}(s)=A+\frac{B s^{2}}{1+C s^{2}}-\frac{D s^{2}}{1+E s^{4}},
$$

with parameter values of $A=1.05151, B=0.191458$, $C=0.254$ 433, $D=0.180708$, and $E=4.036674$ (see Fig. 1). In obtaining these parameters, the $A$-value was constrained to the OPTX value, the $C$-value determined by the PBE constraint for $s \rightarrow \infty(A+B / C=1.804),{ }^{18}$ while the other three parameters had been fitted to minimize the difference be- tween $F_{\text {SSB-sw }}$ and $F_{\text {OPTX }}$ for $s<0.7$ and between $F_{\text {SSB-sw }}$ and $F_{\text {PBEx }}$ for $s>0.9$. It was shown ${ }^{22}$ that this design of the exchange functional reproduces the results of the switched functional and thus combines the best of OPBE with the best of PBE.

Here we report an improvement of the switched functional, where the parameters of the functional have been optimized for a number of different interactions. Moreover, we included Grimme's dispersion correction ${ }^{23,24}$ in order to give good behavior for all weak interactions (especially needed for $\pi-\pi$ stacking).

\section{COMPUTATIONAL DETAILS}

All DFT calculations were performed with a locally adapted version of the Amsterdam Density Functional ${ }^{25,26}$ program developed by Baerends et al. The MOs were expanded in a large uncontracted set of Slater-type orbitals (STOs) (TZ2P, QZ4P) (Ref. 27), which are respectively of triple- $\zeta$ quality augmented by two sets of polarization functions and of quadruple- $\zeta$ quality augmented by four sets of polarization functions. An auxiliary set of $s, p, d, f$, and $g$ STOs was used to fit the molecular density and to represent the Coulomb and exchange potentials accurately in each SCF cycle.

Energies and gradients were calculated using the LDA [Slater exchange and VWN (Ref. 28) correlation] with gradient corrections (GGA) for exchange and correlation included self-consistently for each of the functionals used in this study, i.e., the OPBE functional uses the GGA parts of Handy and Cohen's OPTX (Ref. 20) exchange functional with the PBEc (Ref. 18) correlation functional, PBE (Ref. 18) uses both the exchange PBEx and correlation PBEc GGA functionals, while the SSBx functionals combine the GGA part of Eq. (9) with that of the simplified PBE correlation (sPBEc) functional. ${ }^{21}$ We used here the sPBEc and not the original PBEc because the former gives better results for G2-set atomization energies and hydrogen-bonding interactions. ${ }^{21}$ In the case of the newly developed functionals that include Grimme's dispersion correction, we adapted only the $s_{6}$ factor with which this correction is included and kept all other parameters to their default values.

Geometries were optimized with the QUILD program ${ }^{29}$ using adapted delocalized coordinates ${ }^{30}$ because of its superior geometry optimization performance. In the optimization process of the different functionals, we used the Amoeba routines from Numerical Recipes ${ }^{31}$ for minimization of the sum of mean absolute deviation (MAD) values (see below).

\section{CONSTRUCTION OF NEW FUNCTIONALS}

We previously ${ }^{22}$ designed a new exchange-functional formulation (SSB-Sw) that reproduced the results obtained when switching between OPBE and PBE. That study showed that by a small modification of PBEx its poor performance for spin states and reaction barriers can be improved drastically. However, constraining the parameters to those of OPTX may not be optimal for the newly designed functional so we decided to try to optimize them. In one of our previous studies, ${ }^{21}$ we already discovered that by optimizing the pa- 
TABLE I. Different interaction types taken into account for parameter optimization with their specific weights (see also Ref. 21)

\begin{tabular}{|c|c|c|}
\hline Interaction & Weight & Reference \\
\hline Metal-ring distance in ferrocene & 1.0 & 50 \\
\hline Atomization energies G2-1 set ${ }^{\mathrm{a}}$ & 1.0 & 51 \\
\hline Metal-halogen distances ${ }^{\mathrm{b}}$ & 1.0 & 10,44 \\
\hline Accuracy of geometry for set of small molecules ${ }^{c}$ & 2.0 & $13,42,43$ \\
\hline $\begin{array}{l}\mathrm{S}_{\mathrm{N}} 2 \text {-energetics }\left(P_{E} \text { from Ref. } 15 \text { for reactions }\right. \\
\text { A2-A6) }\end{array}$ & 3.0 & 15 \\
\hline$S_{N} 2$-barriers (for reactions A2-A6 in Ref. 15) ${ }^{d}$ & 3.0 & 15 \\
\hline$\pi-\pi$ stacking energy cytosine dimer & 1.0 & 12,52 \\
\hline O-H distance water molecule & 2.0 & \\
\hline $\mathrm{H}-\mathrm{O}-\mathrm{H}$ angle water molecule & 1.0 & \\
\hline Hydrogen-bonding four dimers ${ }^{\mathrm{e}}$ & 3.0 & 47 \\
\hline
\end{tabular}

${ }^{\mathrm{a} 55 \text { molecules in total. }}$

${ }^{\mathrm{b}}$ Ten distances in total.

${ }^{\mathrm{c}} 28$ bonds in total.

${ }^{\mathrm{d}}$ Seven reactions in total, $P_{E}$ is the average of four energy components involved in the $\mathrm{S}_{\mathrm{N}} 2$ energy profile.

${ }^{\mathrm{e}}$ Ammonia dimer, water dimer, formic acid dimer, and formamide dimer.

rameters for atomic exchange energies, the performance for molecular properties is not optimal. On the other hand, by optimizing the parameters to the atomization energies of the G2-1 set, neither other molecular properties nor atomic properties are satisfactory. Therefore, we decided to optimize the parameters for a number of different interactions such as $\pi$ - $\pi$ stacking, hydrogen bonding, $\mathrm{S}_{\mathrm{N}} 2$ barriers, and accuracy of geometries of organic and organometallic systems. In particular, we determined the MAD from reference values (taken from the literature and usually obtained at high-level $\operatorname{CCSD}(\mathrm{T})$ with large basis sets; see Ref. 21 for details) and multiplied it with a weight specific to each interaction (see Table I). The sum of these weighted MAD values was then minimized for obtaining the parameters of the new density functionals.

At first, we optimized simply the parameters in Eq. (4), which are in fact only three independent ones since we applied both the constraint that $A+B / C=1.804$ (see above), and that $D=B$ in order to reduce the number of parameters to be optimized. This led to good behavior for most interactions but showed an elevated MAD value for $\pi$ - $\pi$ stacking and the G2-1 atomization energies. Because $\pi-\pi$ stacking was found $^{12}$ to be a complicated issue for density functional methods and can easily be circumvented by including Grimme's empirical dispersion correction, ${ }^{23,24}$ we decided to try this first. Indeed, this brought the MAD value for stacking down considerably, from ca. $7 \mathrm{kcal} \mathrm{mol}^{-1}$ to ca. $0.5 \mathrm{kcal} \mathrm{mol}^{-1}$. The MAD value for the G2-set however remained quite elevated (ca. $14-16 \mathrm{kcal} \mathrm{mol}^{-1}$ ).

We then realized that this is reminiscent of the situation of Keal and Tozer's ${ }^{32}$ KT1 functional, which worked very well for NMR parameters, but gave sizeable MAD values for molecular properties and atomization energies. Keal and Tozer thereafter experimented with separate factors for LDA exchange and for LDA correlation contributions (to give their KT2 functional), but here we simply explored mixing in a portion of their gradient correction that is given in Eq. (5):

$$
\Delta E_{\mathrm{KT}}=\gamma \sum_{\sigma} \int d^{3} r \frac{\left|\nabla \rho_{\sigma}\right|^{2}}{\rho_{\sigma}^{4 / 3}+\delta}
$$

In this equation $\sigma$ runs over the spin-up and spin-down densities $\rho_{\sigma}$ and $\gamma$ and $\delta$ are parameters; in the KT1 functional, the values are, respectively, $-0.006(\gamma)$ and $0.1(\delta)$. Note that Eq. (5) does not satisfy the uniform density exchange scaling condition and for finite densities may be viewed as a combined exchange-correlation term. It resembles the leading term in the exchange gradient expansion, with the additional constant $\delta$ introduced to prevent divergence of the potential in asymptotic regions. Now, Eq. (5) can be easily rewritten in a form that resembles our $-D s^{2} /\left(1+E s^{4}\right)$ correction in Eq. (4) [where $u$ is a constant that remains to be determined and $C_{x}$ is the coefficient for LDA exchange in spin-polarized form, see Eqs. (7) and (8)]:

$$
\Delta E_{\mathrm{KT}}=u C_{x} \int d^{3} r \sum_{\sigma} \rho_{\sigma}^{4 / 3} \frac{D s_{\sigma}^{2}}{1+\delta / \rho_{\sigma}^{4 / 3}} .
$$

In order to compare it with our formulation in Eq. (4), it should be kept in mind that Eq. (4) shows only the enhancement factor $F(s)$ over LDA exchange, i.e., the total energy is for LDA and GGA exchange functionals given by

$$
\begin{aligned}
& E_{x, \mathrm{LDA}}=\int d^{3} r \varepsilon_{x}^{\mathrm{unif}}, \\
& \varepsilon_{x}^{\text {unif }}=-A_{x} \rho^{4 / 3} ; \quad A_{x}=\frac{3}{4}\left(\frac{3}{\pi}\right)^{1 / 3}, \\
& E_{x, \mathrm{GGA}}=\int d^{3} r \varepsilon_{x}^{\text {unif }} F(s) .
\end{aligned}
$$

Therefore, the correction term in Eq. (4) for the exchange energy is (note that here it has been rewritten in spinpolarized form because that is how it is usually calculated)

$$
\Delta E_{\mathrm{SSB}-\mathrm{sw}}=v C_{x} \int d^{3} r \sum_{\sigma} \rho_{\sigma}^{4 / 3}\left[\frac{D s_{\sigma}^{2}}{1+E s_{\sigma}^{4}}\right] ; \quad C_{x}=A_{x} \cdot 2^{1 / 3} .
$$

If we ignore for the moment the constants $u$ and $v$, the only difference appears in the denominator that reads $1+E s^{4}$ for our SSB formula and $1+\delta / \rho^{4 / 3}$ for KT. It is interesting to note that both our SSB formula and the KT formula correct the enhancement factor $F(s)$ at low $s$ values. However, in case of KT, the situation is a bit more complicated because the enhancement factor cannot be expressed in terms of $s$ only [see Eq. (6)], contrary to what is the case for GGA exchange functionals.

So far, for our newly constructed functionals we constrained the $D$ parameter to be equal to $B$ [see Eq. (4)], which we can now expand to include the KT formula. That is, we take the combination of the SSB and KT corrections and mix them with $u+v=1$ such that the total effect at low $s$ remains the same. By coincidence, we noticed in our optimization procedure of the parameters that an even better performance 
was observed when multiplying the KT correction with an additional factor $F$. The final formulation of our SSB functionals is therefore given in Eq. (9): ${ }^{33}$

$$
\begin{aligned}
& E_{\mathrm{SSB}}=E_{x, \mathrm{SSB}}+E_{c, \mathrm{SSB}}+E_{\mathrm{disp}}, \\
& E_{x, \mathrm{SSB}}=E_{x, \mathrm{SSB}-1}+E_{\mathrm{SSB}-2}, \\
& E_{x, \text { SSB-1 }}=\int d^{3} r \varepsilon_{x}^{\text {unif }} F_{\text {SSB }-1}(s) \\
& F_{\mathrm{SSB}-1}(s)=A+\frac{B s^{2}}{1+C s^{2}}-\frac{B(1-u) s^{2}}{1+E s^{4}}, \\
& E_{\mathrm{SSB}-2}=u F C_{x} \int d^{3} r \sum_{\sigma} \rho_{\sigma}^{4 / 3} \frac{B s_{\sigma}^{2}}{1+\delta / \rho_{\sigma}^{4 / 3}}, \\
& E_{c, \mathrm{SSB}}=\int d^{3} r \rho\left[\varepsilon_{C}^{\mathrm{unif}}(\rho, \zeta)+H^{\mathrm{sPBE}}(\rho, \zeta, t)\right] \\
& \zeta=\frac{\rho_{\text {up }}-\rho_{\text {down }}}{\rho_{\text {total }}}, \\
& t=\frac{|\nabla \rho|}{2 \phi(\zeta) k_{s} \rho} ; \quad k_{s}=\sqrt{\frac{4\left(3 \pi^{2} \rho\right)^{1 / 3}}{\pi}} ; \\
& \phi(\zeta)=\frac{(1+\zeta)^{2 / 3}+(1-\zeta)^{2 / 3}}{2}, \\
& H^{\mathrm{sPBE}}(\rho, \zeta, t)=\gamma \phi^{3} \ln \left[1+\frac{\beta}{\gamma} t^{2} \frac{1}{1+G t^{2}}\right] \\
& G=\frac{\beta}{\gamma} \frac{1}{\exp \left(\frac{-\varepsilon_{C}^{\text {unif }}}{\gamma \phi^{3}}\right)-1}, \\
& E_{\mathrm{disp}}=-s_{6} \sum_{i=1}^{N-1} \sum_{j=i+1}^{N} \frac{C_{6, i j}}{R_{i j}^{6}} f_{\mathrm{dmp}}\left(R_{i j}\right) \text {; } \\
& f_{\mathrm{dmp}}\left(R_{i j}\right)=1 /\left[1+e^{\alpha-\alpha\left(R_{i j} / R_{\mathrm{ref}}\right)}\right] .
\end{aligned}
$$

We have no reason to adjust the values of $\alpha, \beta, \gamma$, and $\delta$, nor the high $s$ limit of PBE, so we therefore keep these at their original values of $20, \sim 0.066725, \sim 0.031091,0.1$, and 1.804 , respectively. The other parameter values for the SSB and SSB-D functionals are given in Table II. It should be noted that for the construction of the SSB-D functional that includes Grimme's dispersion correction, the $s_{6}$ factor for dispersion correction was included in the parameter set to be optimized and was thus optimized simultaneously with the other ones.

Similar to OPTX and Grimme's B97-D functional, ${ }^{24}$ the SSB functionals do not satisfy the uniform electron gas (UEG) limit at $s=0$. Instead they have elevated values of 1.05151 (OPTX), 1.08662 (B97-D), and 1.079966 (SSBD). It seems that for reliable results in chemistry, factors different from one are needed as also evidenced by, e.g., the
TABLE II. Parameters of new functionals [see Eq. (8)].

\begin{tabular}{llll}
\hline \hline & SSB-sw & SSB & \multicolumn{1}{c}{ SSB-D } \\
\hline$A$ & 1.05151 & 1.071769 & 1.079966 \\
$B$ & 0.191458 & 0.137574 & 0.197465 \\
$C$ & $0.254433^{\mathrm{a}}$ & $0.187883^{\mathrm{a}}$ & $0.272729^{\mathrm{a}}$ \\
$D$ & 0.180708 & $0.137574^{\mathrm{b}}$ & $0.197465^{\mathrm{b}}$ \\
$E$ & 4.036674 & 6.635315 & 5.873645 \\
$u$ & $\ldots$ & -1.205643 & -0.749940 \\
$F$ & $\ldots$ & 0.995010 & 0.949488 \\
${ }^{\mathrm{c}}{ }^{\mathrm{c}}$ & 0.0 & 0.0 & 0.847455 \\
\hline
\end{tabular}

${ }^{\mathrm{a} C o n s t r a i n e d ~ t o ~ f u l f i l l ~} A+B / C=1.804$ (see text).

${ }^{\mathrm{b}}$ Constrained to $D=B$ (see text).

${ }^{\mathrm{c}} s_{6}$ factor of Grimme's dispersion correction.

hybrid functionals B3LYP and TPSSh that have factors of 0.8 and 0.9 , respectively (plus a wildly complicated functional, i.e., portion of HF exchange, that does not depend explicitly on $s$ ). In the design of our SSB-D functional, we aim at reliable results for biological and chemical applications, which seem to dictate that we have to sacrifice the UEG limit.

\section{PERFORMANCE OF NEW FUNCTIONALS}

The inclusion of the $\mathrm{KT}$ correction indeed reduces the MAD value for the atomization energies considerably and is now similar to that of PBE (see Table III), although it remains higher than that of other functionals such as OPBE (see Table III), TPSSh $^{34,35}$ or B3LYP. ${ }^{36}$ This is observed both for the smaller G2-1 (55 molecules) and the larger G2/97 (148 molecules) set. It is probably related to its performance for atomic properties and not as much the molecular ones because for a wide range of molecular properties of biological interest (hydrogen bonding, $\pi$ - $\pi$ stacking, spin-state energies, reaction barriers) our new SSB-D functional performs as well or better than these functionals (see below). It might be that the GGA formulation is simply not flexible enough for satisfying both the G2-set and other properties, as advocated by Perdew et al., ${ }^{37}$ and one should go to the MGGA level for improvement of the atomization energies. Indeed, the PBE atomization energy error is greatly reduced when going to its MGGA counterpart (TPSS). ${ }^{34,35}$ We have not attempted to do that here and will leave it for future studies.

\section{A. Spin-state splittings}

One of the aims of our recent studies was to determine what makes the PBE not work well for spin-state splittings of transition-metal complexes and why OPBE does. Our preliminary study with our SSB-sw functional ${ }^{22}$ indicated that a very small region in $s$ space is responsible for it. Since we did not fit to any spin-state splittings (in contrast with B3LYP*), we were interested to see how the newly developed functional performs in this respect. Given in Table IV are the $\Delta E_{\mathrm{HL}}$ splittings between the low-spin and high-spin states for a number of iron complexes. Note that no experimental or "exact" reference spin-state energies are available for these complexes, but previous studies by one of us have shown the reliability of OPBE. ${ }^{9,10}$ For the first complex 
TABLE III. MAD values for energies and geometry parameters.

\begin{tabular}{|c|c|c|c|c|c|}
\hline Interaction & OPBE & PBE & SSB-sw & SSB & SSB-D \\
\hline Metal-ring ferrocene (pm) & 4.2 & 0.0 & 3.9 & 3.3 & 3.3 \\
\hline Atomization $\mathrm{G} 2$ set $\left(\mathrm{kcal} \mathrm{mol}^{-1}\right)^{\mathrm{a}}$ & $5.3(8.9)$ & $8.5(16.3)$ & $15.4(20.0)$ & $10.3(30.6)$ & $8.7(23.8)$ \\
\hline Metal-halogen distances (pm) & $1.1^{\mathrm{b}}$ & $0.6^{\mathrm{b}}$ & 1.8 & 1.1 & 0.8 \\
\hline Geometry small molecules (pm) & 0.9 & 1.0 & 0.8 & 0.7 & 0.6 \\
\hline $\mathrm{S}_{\mathrm{N}} 2$-energetics $\left(\mathrm{kcal} \mathrm{mol}^{-1}\right)$ & $2.1^{\mathrm{c}}$ & $4.0^{\mathrm{c}}$ & 2.4 & 2.5 & 2.4 \\
\hline $\mathrm{S}_{\mathrm{N}} 2$-barriers $\left(\mathrm{kcal} \mathrm{mol}^{-1}\right)$ & $3.4^{\mathrm{c}}$ & $6.4^{\mathrm{c}}$ & 3.5 & 3.5 & 2.6 \\
\hline$\pi-\pi$ stacking (cytosine $)_{2}\left(\mathrm{kcal} \mathrm{mol}^{-1}\right)$ & 15.6 & 7.1 & 7.1 & 6.8 & 0.1 \\
\hline $\mathrm{O}-\mathrm{H}$ distance water $(\mathrm{pm})$ & 0.5 & 1.3 & 0.4 & 0.1 & 0.1 \\
\hline $\mathrm{H}-\mathrm{O}-\mathrm{H}$ angle water (deg) & 0.9 & 0.3 & 0.6 & 1.1 & 1.1 \\
\hline $\mathrm{H}$-bonding four dimers $(\mathrm{pm})^{\mathrm{d}}$ & 29.3 & 1.7 & 4.7 & 0.4 & 1.2 \\
\hline
\end{tabular}

${ }^{\mathrm{a}}$ Values for G2-1 set, in parentheses values for G2/97 set.

${ }^{\mathrm{b}}$ From Ref. 10

${ }^{c}$ From Ref. 15.

${ }^{\mathrm{d}}$ Ammonia dimer, water dimer, formic acid dimer, and formamide dimer.

$\mathrm{Fe}\left(L_{1}\right)$, we calculated the vertical spin-state splitting and observed both with OPBE and the SSB functionals a high-spin ground-state, which is in accord with experimental data. ${ }^{9,38}$ The PBE functional on the other hand predicts a low-spin state, ${ }^{9}$ already with the high-spin structure in which the ironligand distances are elongated (2.3-2.4 $)$, which disfavors the low-spin state considerably.

The other two iron complexes contain pyridylmethylamine ligands, which are structurally and chemically very similar, yet display different spin states. With the amine (amp) ligand a high-spin ground-state is observed experimentally, while with the diamine (dpa) ligand a low-spin is found. Previously, ${ }^{10}$ it was shown that OPBE is one of very few DFT functionals that is able to distinguish between these two complexes in terms of their spin ground state. To that list we can now add the SSB-D functional, which shows even more favorable spin-state splittings than OPBE with values of $12.6 \mathrm{kcal} \mathrm{mol}^{-1}$ (SSB-D) versus 8.5 (OPBE) for $\mathrm{Fe}(\mathrm{amp})_{2} \mathrm{Cl}_{2}$ and $-5.2 \mathrm{kcal} \mathrm{mol}^{-1}$ versus -2.3 (OPBE) for $\mathrm{Fe}(\mathrm{dpa})_{2}^{2+}$ (see Table IV).

Finally, we included the nickel complex $\mathrm{Ni}(\mathrm{edt})_{2}^{2-}$, which is a model complex for the catalytic cycle of NiFe-hydrogenase. ${ }^{39,40}$ Previous studies showed that, in particular, B3LYP and, to a lesser degree B3LYP*, were unable to predict the low-spin ground-state for this complex. ${ }^{39}$ However, the influence of the basis set is often very important ${ }^{41}$ and also played a role in this case. With a poor Gaussian-type orbital (GTO) basis, B3LYP indeed predicts a (high-spin) triplet ground state, but both with a good GTO basis and with STO basis sets it narrowly $\left(1.0 \mathrm{kcal} \mathrm{mol}^{-1}\right.$ or less $)$ predicts the singlet ground state. ${ }^{40}$ The situation is completely different for OPBE or SSB-D, which correctly predicts the singlet ground state (see Table IV) and with a substantial singlettriplet splitting $\left(6-10 \mathrm{kcal} \mathrm{mol}^{-1}\right)$. In this case, PBE also correctly predicts the ground state but this is not surprising since it favors low-spin state like most early GGA functionals. ${ }^{8}$

\section{B. Nucleophilic substitution reactions}

The other case where OPBE performs remarkably well and PBE does not ${ }^{14}$ is for the reaction barriers of bimolecular nucleophilic substitution $\left(\mathrm{S}_{\mathrm{N}} 2\right)$ reactions (see Scheme 1). These barriers were included in the optimization process of the SSB-D functional and it is therefore no surprise that the functional works well for them. Indeed, it gives the lowest deviation for the central barrier $\left(2.6 \mathrm{kcal} \mathrm{mol}^{-1}\right)$ for any of the GGA functionals studied so far (see Table III). In order to get an independent check on the accuracy for these barriers, we also used our new functionals for a number of systems taken from a recent study by some of us. ${ }^{16}$ In that study, the anti-E2, syn-E2, and $\mathrm{S}_{\mathrm{N}} 2$ pathways of $X^{-}+\mathrm{CH}_{3} \mathrm{CH}_{2} X$ $(X=\mathrm{F}, \mathrm{Cl})$ was investigated with DFT and a number of wave function methods (HF, MPx, coupled cluster). Good overall agreement with the $a b$ initio benchmark data was observed for the OPBE functional, which gives a MAD value for the central barrier of $3.8 \mathrm{kcal} \mathrm{mol}^{-1}$ (see Table V). The MAD value for the PBE functional is almost twice as large (6.6 $\mathrm{kcal} \mathrm{mol}^{-1}$ ), while the SSB-D gives the best performance with a MAD value of $3.5 \mathrm{kcal} \mathrm{mol}^{-1}$ (see Table V).

TABLE IV. Spin-state splittings $\Delta E_{\mathrm{HL}}\left(E_{\mathrm{LS}}-E_{\mathrm{HS}}\right)\left(\mathrm{kcal} \mathrm{mol}^{-1}\right)$ for iron complexes.

\begin{tabular}{lrrrrr}
\hline \hline Compd. & OPBE & PBE & SSB-sw & SSB & SSB-D \\
\hline $\mathrm{Fe}\left(\mathrm{L}_{1}\right)^{\mathrm{a}}$ & 12.4 & -4.1 & 9.4 & 13.5 & 15.6 \\
$\mathrm{Fe}(\mathrm{amp})_{2} \mathrm{Cl}_{2}{ }^{\mathrm{b}}$ & 8.5 & -8.4 & -1.0 & 6.4 & 12.6 \\
$\mathrm{Fe}(\mathrm{dpa})_{2}^{2+\mathrm{c}}$ & -2.3 & -22.1 & -14.9 & -7.1 & -5.2 \\
$\mathrm{Ni}(\mathrm{edt})_{2}^{2-\mathrm{d}}$ & -10.3 & -11.4 & -12.5 & -9.7 & -6.8 \\
\hline \hline
\end{tabular}

${ }^{\mathrm{a}} L_{1}=\left(\mathrm{N}\left(\mathrm{CH}_{2}-\mathrm{o}-\mathrm{C}_{6} \mathrm{H}_{4} \mathrm{~S}\right)_{3}\right)(1$-me-imidazole $)$, high-spin in experiments, see Refs. 9, 10, and 38.

${ }^{b}$ amp=2-pyridylmethylamine, high-spin exp., see Refs. 10 and 53.

${ }^{c} \mathrm{dpa}=\mathrm{di}(2$-pyridylmethyl)amine, low-spin exp., see Refs. 10 and 53.

${ }^{\mathrm{d}}$ edt $=$ ethane-1,2-dithiolate, low-spin exp., see Refs. 39 and 40. 


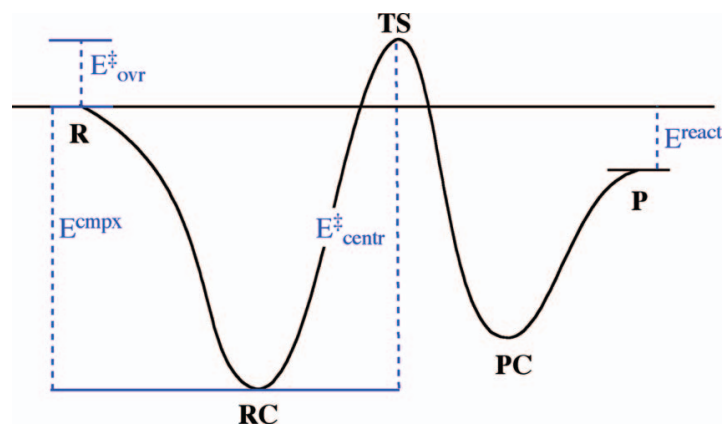

SCHEME 1. Energy profile for $\mathrm{S}_{\mathrm{N}} 2$ reactions.

Previously, we also investigated the geometries of stationary points and compared these with high-level $\operatorname{CCSD}(\mathrm{T})$ geometries. $^{14}$ The best agreement was observed for the OLYP and OPBE functionals, with $P_{G}$ values $\left[P_{G}\right.$ $=\operatorname{MAD}(R) \cdot \operatorname{MAD}(\theta)$, see Ref. 14] of 0.033 and $0.042 \AA$ deg, respectively, which was already significantly better than the best MGGA and hybrid functionals, OLAP3 and mPBEOKCIS, which showed values 0.060 and $0.068 \AA$ deg, respectively. ${ }^{14}$ Our new SSB-D functional improves upon all of them with a value of $0.014 \AA \mathrm{deg}$ (see Table V).

\section{Accuracy of geometries of small molecules and metal compounds}

The good performance of our new functional is corroborated by good performance regarding geometry parameters for two sets of molecules. The first one comprises a set of 19 small (first row) molecules that was previously studied by Helgaker and co-workers ${ }^{42,43}$ with a number of high-level $a b$ initio methods and correlation-consistent basis sets, and by one of us for DFT functionals. ${ }^{13}$ The best performance was observed with $\operatorname{CCSD}(\mathrm{T})$ with an average absolute deviation from experimental data of less than $0.2 \mathrm{pm}$. In fact they predicted that one experimental value was incorrect, which was confirmed by a subsequent experimental study. ${ }^{43}$ Density functional methods showed in general good agreement as well, with MAD values of around $1 \mathrm{pm} .{ }^{13}$ The PBE and OPBE functionals were found to be one of the best DFT functionals for this set with MAD values of ca. 0.9 pm. ${ }^{13,14}$ Our new SSB-D functional significantly improves upon them with a MAD value of $0.6 \mathrm{pm}$. (see Table III).

Bühl and co-workers ${ }^{44-46}$ recently made a number of benchmarks for transition-metal complexes. In their first paper, they studied a diverse set of 32 molecules and also a more compact set of metal halides. ${ }^{44}$ The performance of the DFT functionals for the latter compact set was found to be representative of the performance for the larger set. Therefore, we also studied this set with the new functional and found good behavior, with a MAD value of $0.8 \mathrm{pm}$ that is between that of OPBE and PBE (see Table III). Note that the value of $1.1 \mathrm{pm}$ for OPBE was already improving upon those of B3LYP and B3LYP*, ${ }^{10}$ while the SSB-D value is similar to the best functional (TPSS) in the study of Bühl and Kabrede. $^{44}$

\section{Weak interactions}

An important interaction for the study of the chemistry of biological systems is apart from the spin-state splittings (when transition metals are present) also weak interactions such as hydrogen bonding and $\pi$ - $\pi$ interactions. Since these interactions are much weaker than the strong intramolecular interactions, it is more difficult to obtain them correctly. This holds not only for DFT but also for wave function-based methods where the basis set superposition error (BSSE) is often of the same order of magnitude as the interaction itself, especially when using small or medium-sized GTO basis sets.

In our design of the SSB-D functional, we included both hydrogen bonding and $\pi$ - $\pi$ stacking systems in the optimization process, which helped to get performance for both. For the hydrogen bonding in four dimers (ammonia, water, formic acid, formamide), we showed previously ${ }^{22}$ that PBE performs very well, while OPBE significantly underestimates the hydrogen-bonding energies. This is also reflected in the hydrogen-bond distances of the same four dimers, where

TABLE V. MAD values in nucleophilic substitution energetics $\left(\mathrm{kcal} \mathrm{mol}^{-1}\right.$; see Scheme 1) and geometries (pm, deg).

\begin{tabular}{|c|c|c|c|c|c|}
\hline & $\mathrm{OPBE}^{\mathrm{a}}$ & $\mathrm{PBE}^{\mathrm{a}}$ & SSB-sw & SSB & SSB-D \\
\hline \multicolumn{6}{|c|}{$S_{N} 2$ reactions from Ref. 15} \\
\hline$\Delta E^{\mathrm{cmpx}}$ & 3.5 & 1.5 & 1.5 & 1.7 & 2.1 \\
\hline$\Delta E^{\ddagger \text {,centr }}$ & 3.4 & 6.4 & 3.5 & 3.5 & 2.6 \\
\hline$\Delta E^{\ddagger, \mathrm{ovr}}$ & 1.3 & 7.8 & 4.3 & 4.6 & 4.7 \\
\hline$\Delta E^{\text {react }}$ & 0.3 & 0.3 & 0.3 & 0.3 & 0.3 \\
\hline$P_{E}^{\mathrm{b}}$ & 2.1 & 4.0 & 2.4 & 2.5 & 2.4 \\
\hline$R_{\text {all }}$ & 6.3 & 12.0 & 2.3 & 2.4 & 1.9 \\
\hline$\theta_{\text {all }}$ & 0.7 & 5.1 & 0.8 & 0.7 & 0.8 \\
\hline$P_{G}^{c}$ & 0.04 & 0.61 & 0.02 & 0.02 & 0.01 \\
\hline \multicolumn{6}{|c|}{$\mathrm{E} 2$ and $\mathrm{S}_{\mathrm{N}} 2$ reactions from Ref. $16^{\mathrm{d}}$} \\
\hline$\Delta E^{\ddagger \text {,centr }}$ & 3.8 & 6.6 & 4.2 & 3.7 & 3.5 \\
\hline
\end{tabular}


TABLE VI. Energies $\left(\mathrm{kcal} \mathrm{mol}^{-1}\right)$ and distances (pm) for weak interactions using TZ2P STO basis.

\begin{tabular}{|c|c|c|c|c|c|}
\hline & OPBE & PBE & SSB-sw & SSB & SSB-D \\
\hline \multicolumn{6}{|c|}{$\mathrm{S} 22 \operatorname{set}^{\mathrm{a}}$} \\
\hline $\mathrm{MAD}^{\mathrm{b}}$ & $8.1(4.1)^{\mathrm{c}}$ & $2.8(0.6)^{\mathrm{d}}$ & 3.3 & 2.7 & 0.5 \\
\hline \multicolumn{6}{|c|}{ A:T base pair } \\
\hline$R(\mathrm{~N} 6-\mathrm{O} 4)$ & $299.2(289.8)^{\mathrm{c}}$ & $287.3(285.2)^{\mathrm{d}}$ & 284.4 & 286.7 & 287.6 \\
\hline$R(\mathrm{~N} 1-\mathrm{N} 3)$ & $293.5(282.3)^{c}$ & $280.2(275.1)^{\mathrm{d}}$ & 277.7 & 285.1 & 279.1 \\
\hline$\Delta E_{\mathrm{tot}}$ & $-5.5(-10.0)^{\mathrm{c}}$ & $-13.9(-17.3)^{\mathrm{d}}$ & -13.8 & -13.2 & -15.6 \\
\hline \multicolumn{6}{|c|}{$\mathrm{G}: \mathrm{C}$ base pair } \\
\hline$R(\mathrm{O} 6-\mathrm{N} 4)$ & $280.2(273.7)^{\mathrm{c}}$ & $273.4(270.9)^{\mathrm{d}}$ & 271.7 & 275.9 & 277.0 \\
\hline$R(\mathrm{~N} 1-\mathrm{N} 3)$ & $297.5(289.9)^{\mathrm{c}}$ & $288.7(285.7)^{\mathrm{d}}$ & 287.6 & 288.9 & 288.4 \\
\hline$R(\mathrm{~N} 2-\mathrm{O} 2)$ & $297.7(290.0)^{\mathrm{c}}$ & $287.2(284.5)^{\mathrm{d}}$ & 285.4 & 284.1 & 283.9 \\
\hline$\Delta E_{\mathrm{tot}}$ & $-15.4(-21.6)^{\mathrm{c}}$ & $-26.9(-31.3)^{\mathrm{d}}$ & -27.4 & -26.3 & -28.3 \\
\hline MAD $R^{\mathrm{e}}$ & $8.8(1.4)^{\mathrm{c}}$ & $1.9(4.5)^{\mathrm{d}}$ & 3.4 & 1.8 & 2.9 \\
\hline MAD $\Delta E^{\mathrm{e}}$ & $10.6(6.0)^{\mathrm{c}}$ & $1.2(2.5)^{\mathrm{d}}$ & 1.2 & 2.1 & 0.1 \\
\hline \multicolumn{6}{|c|}{$(\text { anthracene })_{2}$ dissociation ${ }^{\mathrm{f}}$} \\
\hline$\Delta E(2 \cdot 1-2)$ & $-15.4(5.4)^{\mathrm{c}, \mathrm{g}}$ & $-12.6(3.0)^{\mathrm{d}, \mathrm{g}}$ & $-2.0^{\mathrm{g}}$ & $0.0^{\mathrm{g}}$ & 10.6 \\
\hline \multicolumn{6}{|c|}{ Branching $^{\mathrm{h}}$} \\
\hline$\Delta E(3 \mathrm{~b}-3 \mathrm{a})$ & $-13.5(-3.0)^{\mathrm{c}}$ & $-5.1(2.7)^{\mathrm{d}}$ & -5.5 & -5.0 & 2.1 \\
\hline \multicolumn{6}{|c|}{ 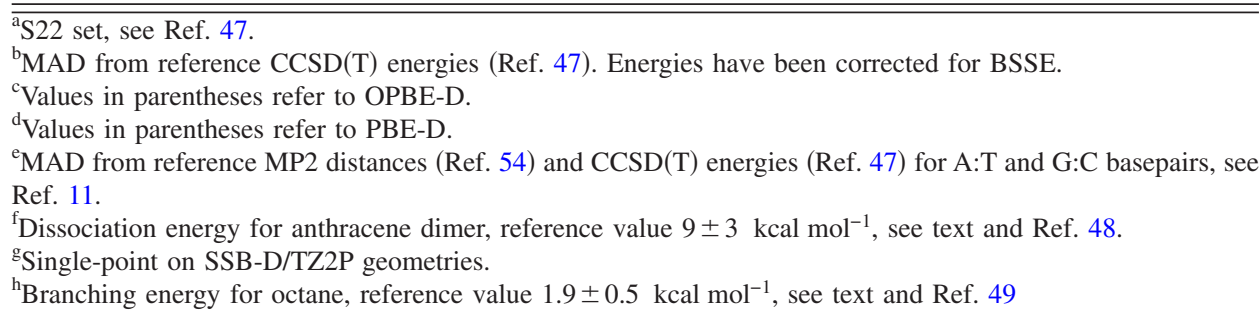 } \\
\hline
\end{tabular}

OPBE shows a deviation of $29.3 \mathrm{pm}$ from reference $\operatorname{CCSD}(\mathrm{T}) \mathrm{data}^{47}$ while PBE gives a deviation of only $1.7 \mathrm{pm}$ (see Table III). SSB-D further improves upon them with a value of only $1.2 \mathrm{pm}$.

For the stacking interaction we included the antiparallel cytosine dimer, which has an interaction energy of -9.9 $\mathrm{kcal} \mathrm{mol}^{-1}$ with $\operatorname{CCSD}(\mathrm{T})$. PBE underestimates this interaction considerably $\left(-2.8 \mathrm{kcal} \mathrm{mol}^{-1}\right.$ and hence a MAD value of $7.1 \mathrm{kcal} \mathrm{mol}^{-1}$, see Table III), while OPBE even shows repulsion instead of attraction (MAD value $15.6 \mathrm{kcal} \mathrm{mol}^{-1}$ ). Our new SSB-D again performs very well with a MAD value of only $0.1 \mathrm{kcal} \mathrm{mol}^{-1}$.

Hobza and co-workers ${ }^{47}$ designed a benchmark set of 22 weakly bound molecules (S22) with high-level reference data with which other methods can be compared. The poor performance of OPBE for weak interactions is well reflected in its MAD value for this S22 set $\left(8.1 \mathrm{kcal} \mathrm{mol}^{-1}\right.$, see Table $\mathrm{VI})$, while the MAD value of PBE $\left(2.8 \mathrm{kcal} \mathrm{mol}^{-1}\right)$ reflects both its better hydrogen-bonding capacities as well as its poorer performance for $\pi-\pi$ stacking. This is confirmed by the PBE-D data (including Grimme's dispersion correction to improve $\pi-\pi$ stacking) for the S22 set that improves considerably $\left(0.6 \mathrm{kcal} \mathrm{mol}^{-1}\right)$, while the OPBE-D data (where we used a $s_{6}$ value of 1.0) remain poor. Our new SSB-D that works well for both $\mathrm{H}$-bonding and $\pi-\pi$ stacking gives a very good performance for this S22 set, with a MAD value of only $0.5 \mathrm{kcal} \mathrm{mol}^{-1}$. Note that our SSB-D energetic data have been corrected for BSSE (even though with STO basis sets, this correction is small, i.e., $0.5 \mathrm{kcal} \mathrm{mol}^{-1}$ or less) in spite of Grimme's proposal ${ }^{24}$ to avoid doing so when sufficiently large (polarized triple-zeta valence or larger) basis sets are used. Since the dispersion correction does not involve basis sets, the BSSE will affect only the other energy terms just as in regular DFT functionals without the dispersion correction.

The good performance of SSB-D for weak interactions is further shown for the hydrogen-bonding patterns of DNA and RNA base pairs A:T and G:C. Recently, ${ }^{11}$ we compared the performance of a number of density functionals with STO and GTO basis sets and found good performance for either BP86, PBE, or PW91. Note that PBE-D overbinds and results in a deterioration compared to the PBE data (see Table VI). Our new SSB-D functional performs very well, with a MAD value with respect to the $\operatorname{CCSD}(\mathrm{T})$ energies ${ }^{47}$ of only $0.1 \mathrm{kcal} \mathrm{mol}^{-1}$ (see Table VI).

Grimme reported recently a study ${ }^{48}$ on the importance of intramolecular and intermolecular van der Waals interactions for organic reactions by looking at the dimerization of anthracene (see Scheme 2). High-level ab initio data at SCSMP2, QCISD(T), and diffusion Monte Carlo (DMC) indicated that this dissociation is endothermic, i.e., the dimer is more stable than two infinitely separated monomers. The en-
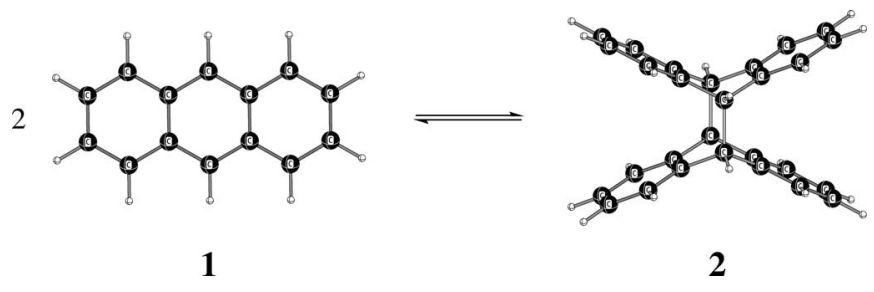

SCHEME 2. Dimerization of anthracene (1). 


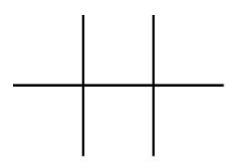

3a

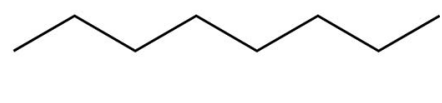

$\mathbf{3 b}$

SCHEME 3. Branched (3a) vs linear (3b) octane.

ergy associated with this dissociation was estimated at $+9 \pm 3 \mathrm{kcal} \mathrm{mol}^{-1}$, based on the predictions by DMC of +9 $\mathrm{kcal} \mathrm{mol}^{-1}$, QCISD(T) with +9.5 $\mathrm{kcal} \mathrm{mol}^{-1}$, and SCSMP2 with $+13.4 \mathrm{kcal} \mathrm{mol}^{-1}$. ${ }^{48}$ A number of DFT functionals was also tested, but with disappointing behavior: B3LYP gave a value of $-26 \mathrm{kcal} \mathrm{mol}^{-1}, \mathrm{PBE}$ gave -15 , and its dispersion-corrected counterpart (PBE-D) gave -4 $\mathrm{kcal} \mathrm{mol}^{-1}$ (data from Ref. 48, which are slightly different from our values in Table VI because of the different basis set). Note that all these three functionals resulted in exothermic dissociation. We also studied this process at the SSB-D/ TZ2P level and found in contrast an endothermic dissociation of $10.6 \mathrm{kcal} \mathrm{mol}^{-1}$. Not only is the sign correct, the energy value is also in perfect agreement with the DMC and QCISD(T) data. $^{48}$

In another recent study, Grimme reported another failure of density functionals, this time for the stereoelectronic effects in alkane isomers. ${ }^{49}$ The difference in stability of branched versus linear alkanes was with density functionals found to be systematically in favor of the linear alkanes. In contrast, experiment, MP2, and SCS-MP2 indicated that the branched alkanes should be more favorable. In the case of octane, the difference in energy should be $1.9 \pm 0.5 \mathrm{kcal} \mathrm{mol}^{-1}$ in favor of branched octane [3(a), see Scheme 3], ${ }^{49}$ which is well reproduced with SCS-MP2 that gives a value of $1.4 \mathrm{kcal} \mathrm{mol}^{-1}$. Density functionals on the other hand showed a systematic preference in favor of linear octane [3(b), see Scheme 3] with values ranging from -3.5 (B2PLYP) to -9.9 (BLYP) $\mathrm{kcal} \mathrm{mol}^{-1}$. Previous studies speculated that this might be due to the failure of density functionals for van der Waals interactions, but this was found to play only a secondary role (e.g., BLYP-D still gave a value of $\left.-5.5 \mathrm{kcal} \mathrm{mol}^{-1}\right)$. Instead, Grimme attributed the failure of density functionals to the neglect of medium-range electron correlation effects and strongly suggested that the energy for the 3 (a) $\rightarrow 3$ (b) reaction be mandatory for checking newly developed density functionals. ${ }^{49}$ We therefore studied these two molecules and found indeed for PBE and OPBE the anticipated preference for linear alkanes (see Table VI). However, our SSB-D functional does not fail at all, but instead indicates a clear preference for the branched octane structure as it should. Moreover, the energy for the range electron correlation effects and strongly suggested that the energy for the $3(\mathrm{a}) \rightarrow 3(\mathrm{~b})$ reaction $\left(2.1 \mathrm{kcal} \mathrm{mol}^{-1}\right.$ at SSB-D/TZ2P, see Table VI) is in excellent agreement with the reference data of $1.9 \mathrm{kcal} \mathrm{mol}^{-1}$.

\section{CONCLUSIONS}

Based on previous studies where we investigated what governs the behavior of OPBE and PBE for spin states and $\mathrm{S}_{\mathrm{N}} 2$ reaction barriers, we designed a new DFT functional that mixes the best of both for these interactions. Together with Grimme's dispersion correction we have thus constructed a new density functional that is based on a small correction of the nonempirical PBE exchange functional. Moreover, our new SSB-D functional partly contains the gradient expansion functional by Keal and Tozer, which could easily be rewritten in a form similar to our small correction. The combination of our small correction and the gradient expansion functional affects only a small region in the reduced gradient $s$ space, but has a large influence on the performance of the functional. The SSB-D functional is shown to work well for spin states of transition-metal complexes, $\mathrm{S}_{\mathrm{N}} 2$ reaction barriers, accuracy of geometries, hydrogen bonding, and $\pi-\pi$ stacking interactions. We tested the new functional on a number of standard test sets such as Hobza's S22 set for weak interactions for which we find a MAD of $0.46 \mathrm{kcal} \mathrm{mol}^{-1}$. Moreover, we investigated two systems that had been brought forward by Grimme as difficult cases and for which density functional methods were shown to fail dramatically. For both the dissociation of the anthracene dimer as for the branching energy of octane do we observe excellent behavior. Not only do we predict the correct sign for these interactions, our energies of $+10.6 \mathrm{kcal} \mathrm{mol}^{-1}$ for the former and $+2.1 \mathrm{kcal} \mathrm{mol}^{-1}$ for the latter are in excellent agreement with SCS-MP2 and DMC data and experiment. We therefore show that it is possible to get the correct energetics for these difficult cases also with a density functional method, at least if SSB-D is used.

\section{ACKNOWLEDGMENTS}

We thank the HPC-Europa program of the European Union, the Netherlands Organization for Scientific Research (NWO), the DURSI Project Nos. 2009SGR528 and 2009SGR637, and the Spanish MEC (Project Nos. CTQ2008-03077/BQU and CTQ2008-06532/BQU) for financial support.

${ }^{1}$ W. Koch and M. C. Holthausen, A Chemist's Guide to Density Functional Theory (Wiley-VCH, Weinheim, 2000).

${ }^{2}$ R. Dreizler and E. Gross, Density Functional Theory (Plenum, New York, 1995).

${ }^{3}$ R. G. Parr and W. Yang, Density Functional Theory of Atoms and Molecules (Oxford University Press, New York, 1989).

${ }^{4}$ J. P. Perdew and W. Yue, Phys. Rev. B 33, 8800 (1986).

${ }^{5}$ J. P. Perdew and L. A. Constantin, Phys. Rev. B 75, 155109 (2007).

${ }^{6}$ J. P. Perdew, J. M. Tao, V. N. Staroverov, and G. E. Scuseria, J. Chem. Phys. 120, 6898 (2004).

${ }^{7}$ J. P. Perdew, A. Ruzsinszky, J. M. Tao, V. N. Staroverov, G. E. Scuseria, and G. I. Csonka, J. Chem. Phys. 123, 062201 (2005).

${ }^{8}$ J. P. Perdew, A. Ruzsinszky, L. A. Constantin, J. Sun, and G. I. Csonka, J. Chem. Theory Comput. 5, 902 (2009).

${ }^{9}$ M. Swart, A. R. Groenhof, A. W. Ehlers, and K. Lammertsma, J. Phys. Chem. A 108, 5479 (2004).

${ }^{10}$ M. Swart, J. Chem. Theory Comput. 4, 2057 (2008).

${ }^{11}$ T. van der Wijst, C. Fonseca Guerra, M. Swart, and F. M. Bickelhaupt, Chem. Phys. Lett. 426, 415 (2006).

${ }^{12}$ M. Swart, T. van der Wijst, C. Fonseca Guerra, and F. M. Bickelhaupt, J. Mol. Model. 13, 1245 (2007).

${ }^{13}$ M. Swart and J. G. Snijders, Theor. Chem. Acc. 110, 34 (2003).

${ }^{14}$ M. Swart, A. W. Ehlers, and K. Lammertsma, Mol. Phys. 102, 2467 (2004).

${ }^{15}$ M. Swart, M. Solà, and F. M. Bickelhaupt, J. Comput. Chem. 28, 1551 (2007).

${ }^{16}$ A. P. Bento, M. Solà, and F. M. Bickelhaupt, J. Chem. Theory Comput. 
4, 929 (2008).

${ }^{17}$ A. P. Bento, M. Solà, and F. M. Bickelhaupt, J. Comput. Chem. 26, 1497 (2005).

${ }^{18}$ J. P. Perdew, K. Burke, and M. Ernzerhof, Phys. Rev. Lett. 77, 3865 (1996).

${ }^{19}$ J. P. Perdew, K. Burke, and Y. Wang, Phys. Rev. B 54, 16533 (1996).

${ }^{20}$ N. C. Handy and A. J. Cohen, Mol. Phys. 99, 403 (2001).

${ }^{21}$ M. Swart, M. Solà, and F. M. Bickelhaupt, in Handbook of Computational Chemistry Research, edited by F. Columbus (Nova Science, Hauppauge, USA, 2009).

${ }^{22}$ M. Swart, M. Solà, and F. M. Bickelhaupt, J. Comp. Meth. Sci. Engin. 9, 69 (2009).

${ }^{23}$ S. Grimme, J. Comput. Chem. 25, 1463 (2004).

${ }^{24}$ S. Grimme, J. Comput. Chem. 27, 1787 (2006).

${ }^{25}$ E. J. Baerends, J. Autschbach, A. Bérces, J. A. Berger, F. M. Bickelhaupt, C. Bo, P. L. de Boeij, P. M. Boerrigter, L. Cavallo, D. P. Chong, L. Deng, R. M. Dickson, D. E. Ellis, M. van Faassen, L. Fan, T. H. Fischer, C. Fonseca Guerra, S. J. A. van Gisbergen, J. A. Groeneveld, O. V. Gritsenko, M. Grüning, F. E. Harris, P. van den Hoek, C. R. Jacob, H. Jacobsen, L. Jensen, E. S. Kadantsev, G. van Kessel, R. Klooster, F. Kootstra, E. van Lenthe, D. A. McCormack, A. Michalak, J. Neugebauer, V. P. Nicu, V. P. Osinga, S. Patchkovskii, P. H. T. Philipsen, D. Post, C. C. Pye, W. Ravenek, P. Romaniello, P. Ros, P. R. T. Schipper, G. Schreckenbach, J. G. Snijders, M. Solà, M. Swart, D. Swerhone, G. te Velde, P. Vernooijs, L. Versluis, L. Visscher, O. Visser, F. Wang, T. A. Wesolowski, E. M. van Wezenbeek, G. Wiesenekker, S. K. Wolff, T. K. Woo, A. L. Yakovlev, and T. Ziegler, ADF 2007.01, SCM, Amsterdam, The Netherlands, 2007.

${ }^{26}$ G. te Velde, F. M. Bickelhaupt, E. J. Baerends, C. Fonseca Guerra, S. J. A. van Gisbergen, J. G. Snijders, and T. Ziegler, J. Comput. Chem. 22 931 (2001).

${ }^{27}$ E. van Lenthe and E. J. Baerends, J. Comput. Chem. 24, 1142 (2003).

${ }^{28}$ S. H. Vosko, L. Wilk, and M. Nusair, Can. J. Phys. 58, 1200 (1980).

${ }^{29}$ M. Swart and F. M. Bickelhaupt, J. Comput. Chem. 29, 724 (2008).

${ }^{30}$ M. Swart and F. M. Bickelhaupt, Int. J. Quantum Chem. 106, 2536 (2006).

${ }^{31}$ W. H. Press, B. P. Flannery, S. A. Teukolsky, and W. T. Vetterling, $N u-$ merical Recipes, The Art of Scientific Computing (Cambridge University Press, Cambridge, 1986).

${ }^{32}$ T. W. Keal and D. J. Tozer, J. Chem. Phys. 119, 3015 (2003).

${ }^{33}$ M. Swart, M. Solà, and F. M. Bickelhaupt, source code for SSB functionals is available at http://iqc.udg.edu/ marcel/ssb, 2009.
${ }^{34}$ J. M. Tao, J. P. Perdew, V. N. Staroverov, and G. E. Scuseria, Phys. Rev. Lett. 91, 146401 (2003).

${ }^{35}$ V. N. Staroverov, G. E. Scuseria, J. Tao, and J. P. Perdew, J. Chem. Phys. 119, 12129 (2003).

${ }^{36}$ P. J. Stephens, F. J. Devlin, C. F. Chabalowski, and M. J. Frisch, J. Phys. Chem. 98, 11623 (1994).

${ }^{37}$ J. P. Perdew, A. Ruzsinszky, G. I. Csonka, O. A. Vydrov, G. E. Scuseria, L. A. Constantin, X. Zhou, and K. Burke, Phys. Rev. Lett. 100, 136406 (2008).

${ }^{38}$ N. Govindaswamy, D. A. Quarless, and S. A. Koch, J. Am. Chem. Soc. 117, 8468 (1995)

${ }^{39}$ M. Bruschi, L. De Gioia, G. Zampella, M. Reiher, P. Fantucci, and M. Stein, J. Biol. Inorg. Chem. 9, 873 (2004).

${ }^{40}$ M. Swart, M. Güell, and M. Solà, in Quantum Biochemistry: Electronic Structure and Biological Activity, edited by C. F. Matta (Wiley, New York, 2010).

${ }^{41}$ M. Güell, J. M. Luis, M. Solà, and M. Swart, J. Phys. Chem. A 112, 6384 (2008).

${ }^{42}$ T. Helgaker, J. Gauss, P. Jørgensen, and J. Olsen, J. Chem. Phys. 106, 6430 (1997).

${ }^{43}$ K. L. Bak, J. Gauss, P. Jørgensen, J. Olsen, T. Helgaker, and J. F. Stanton, J. Chem. Phys. 114, 6548 (2001).

${ }^{44}$ M. Bühl and H. Kabrede, J. Chem. Theory Comput. 2, 1282 (2006).

${ }^{45}$ M. P. Waller, H. Braun, N. Hojdis, and M. Bühl, J. Chem. Theory Comput. 3, 2234 (2007).

${ }^{46}$ M. Bühl, C. Reimann, D. A. Pantazis, T. Bredow, and F. Neese, J. Chem. Theory Comput. 4, 1449 (2008).

${ }^{47}$ P. Jurecka, J. Sponer, J. Cerny, and P. Hobza, Phys. Chem. Chem. Phys. 8, 1985 (2006)

${ }^{48}$ S. Grimme, C. Diedrich, and M. Korth, Angew. Chem. Int. Ed. 45, 625 (2006).

${ }^{49}$ S. Grimme, Angew. Chem. Int. Ed. 45, 4460 (2006).

${ }^{50}$ S. Coriani, A. Haaland, T. Helgaker, and P. Jørgensen, ChemPhysChem 7, 245 (2006)

${ }^{51}$ L. A. Curtiss, K. Raghavachari, G. W. Trucks, and J. A. Pople, J. Chem. Phys. 94, 7221 (1991).

${ }^{52}$ P. Jurecka, J. Sponer, and P. Hobza, J. Phys. Chem. B 108, 5466 (2004).

${ }^{53}$ A. Malassa, H. Görls, A. Buchholz, W. Plass, and M. Westerhausen, Z. Anorg. Allg. Chem. 632, 2355 (2006).

${ }^{54}$ J. Sponer, P. Jurecka, and P. Hobza, J. Am. Chem. Soc. 126, 10142 (2004). 